\title{
Research on the Effect of Backpack With Anti Gravity System Design on Relieving Users' Perceived Exertion
}

\author{
Xi Yang ${ }^{1, *}$ Zhiguan Huang ${ }^{1}$ Pak-To Ng ${ }^{2}$ Wai-Fan Kwan ${ }^{2}$ Yuhe $\mathrm{Li}^{1}$
}

\author{
${ }^{1}$ Guangdong Provincial Engineering Technology Research Center for Sports Assistive Device, Guangzhou Sport \\ University, Guangzhou, Guangdong 510500, China \\ ${ }^{2}$ FX Creations International Ltd., Hong Kong, China \\ *Corresponding author.
}

\begin{abstract}
The mechanism of Anti Gravity System (AGS) backpack lies in the use of highly elastic shoulder straps to create a bouncing motion when the user walks, thus the user doesn't feel the constant load of the bag, which is similar to the ancient Chinese folk transporting tool "BianDan" in work principle. Theoretically speaking, under the same load, this results in a feeling of reduced exertion to users as compared to ordinary backpack. In this paper, a comparative study was made to test the effect of AGS backpack on relieving perceived exertion to users. Two trials were conducted to respectively record the scores of perceived exertion reported by 10 healthy male subjects carrying a loaded AGS backpack and an ordinary backpack on their backs under walking state. The variations of subjective fatigue scores in the 30-minute weight-bearing walking process were compared between two trials. The results showed that for 3 out of 10 subjects, the variation of perceived exertion caused by different types of backpacks were significantly different $(\mathbf{P}<\mathbf{0 . 0 5})$. AGS backpack group also displayed a slower growth of mean perceived exertion score and a decreasing tendency of standard deviation. It was concluded that given the condition of appropriate weight-bearing and walking speed, the backpack with AGS design could give users a labor-saving feeling and more regular growth of subjective fatigue compared with ordinary backpack.
\end{abstract}

Keywords: Anti Gravity System, backpack, highly elastic shoulder straps, Borg rating of perceived exertion scale

\section{INTRODUCTION}

Backpack is an indispensable tool in the daily life of people. An overloaded backpack can cause muscle tension at both sides of spine. A long-time walking of weight-bearing would usually lead to tiredness and discomfort. The design of the backpack is one essential factor that decides the comfort level and the development of physical fatigue. A lot of effort has been done to optimize the design of backpacks to regulate body postures, relieve fatigue and increase comfort. For example, the Active Suspension System developed by Gregory Company consists of Adjust-A-Cant ${ }^{\mathrm{TM}}$ belt, Auto-Cant ${ }^{\mathrm{TM}}$ straps, Foam-X backrest and frame made of aeronautical aluminium alloy, based on which the backpack can automatically rotate to an appropriate degree to better fit to user's body and the weight of backpack can evenly distribute on user's back and waist [1]. The 3D Tepex Suspension System developed by Granite Gear Company, featuring in extremely soft material used at the waist area, is capable of absorbing vibration generated during walking. The design of the rigid top can transfer the weight center of the load to the user's back instead of the neck, while the half-rigid middle part makes the weight evenly distribute on the user's back and the bottom part makes the weight evenly distribute on both sides by naturally surrounding the user above his or her hip. The whole system can alleviate the pressure on user's waist both horizontally and vertically by automatically fitting the spinal curve of the user [2]. Briefly, the improvement of backpack aims at better performance on stability, stickiness between the bag and the back, ventilation and labor saving. The approaches mainly include the new designs of back devices (support device, ventilation device and regulating device) and backpack components (shoulder straps, chest belt, waist belt, shoulder force-bearing straps, bottom force-bearing belt, frame and back cushion) and also the application of new materials on the components. With the continual emergence of new backpack products that claim their advanced design and effects on relieving fatigue and reducing energy cost, testing and assessing their actual effect appears to be a necessary research topic. 
Anti Gravity System (AGS) is a patented technology developed by FX Creations Enterprises Limited to help ease user's carrying burdens. The mechanism of AGS is to create a periodic bouncing reaction as the user walks, thus the user doesn't feel the constant load of the backpack. In theory, a slower progression of perceived exertion can be detected from users carrying AGS backpack. The purpose of the study is to investigate the effect of AGS backpack on relieving user's perceived exertion. Comparative experiments, in which subjects respectively carrying a loaded AGS backpack and an ordinary backpack $(5 \mathrm{~kg})$ on their backs walk at a constant speed for 30 minutes on the treadmill, have been carried out. During the weightbearing walking, the scores of perceived exertion reported by test recipients were recorded every five minutes. The variations of the scores over time were then compared between two trials.

\section{MATERIALS AND METHODS}

\section{A. The mechanism of Anti Gravity System}

Anti Gravity System (AGS ${ }^{\mathrm{TM}}$ ) is inspired by ancient Chinese transporting tool "BianDan" — a shoulder pole made of bamboo that features high strength, elasticity, bending toughness and moderate rigidity. Heavy loads were hung on both ends of "BianDan" and its middle part was put on the porter's shoulder. "BianDan" works as an elastomer when it is load-bearing and deformed. When the porter began to walk, the moving up and down of porter's shoulder would excite the "sping-mass" system composed of "Biandan" and the heavy load, resulting in forced vibration [3]. Compared with the constant static load, human body is more suitable for dynamic load that changes periodically [3] [4]. Though the peak value of pressure exerted by dynamic load is larger than that exerted by static load, more than half of the time in each cycle the pressure exerted by the former is smaller than that exerted by the latter. Consequently, the body can relax and rest during the majority moment of one period. In the carrying method of "BianDan", the relaxation phase is just used to move forward. This can be considered as an important reason for people to feel "labor-saving" when carrying burdens [3] [4] [5].

Similarly, the mechanism of AGS backpack lies in that it can create a bouncing motion during the process of walking so that the user doesn't feel the constant load of the bag but has a feeling of less pressure from the weight on the shoulders. The key technologies of AGS include straps made of ultra bounce cord, hip belt made of patented mesh material Tri-breath ${ }^{\mathrm{TM}}$, and the bag's back consisting of an " $\mathrm{i}$ " support design and a thick cushioning ("Fig. 1")[6]. Ultra bounce is an extra-strength elastic cord. The shoulder straps made of ultra bounce is the core technology of AGS backpack. They serve as a spring in the forced vibration and create a bouncing reaction as the user walks, which counteracts downward gravity force by the bag's upward bounce motion. The up and down motion helps to reduce the pressure on shoulder at different intervals, and the heavier the load or the faster one walks, the bounce reaction intensifies. The hip belt helps stabilize the load around the hip. It is made of a patented mesh material Tri-breath ${ }^{\mathrm{TM}}$, which is shock absorbing and water proof. The bag's back adopts "i" support design to connect the bag to the shoulder straps. It contains a high density polyethylene board to keep the bag body straight and prevent the bag from dropping over the heavy loads. The thick cushioning on the bag's back forms a ridge in the middle to allow ventilation and distribute the pressure evenly, and by this means to provide space for the user's spine [7].

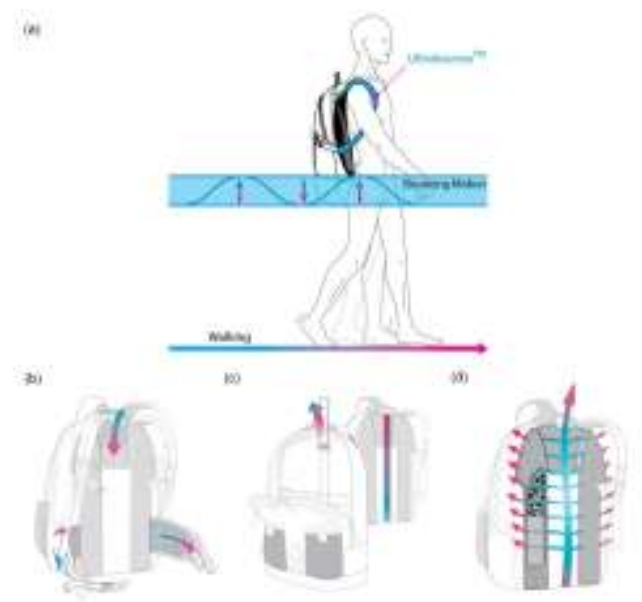

Fig. 1. Mechanism and design of anti gravity system backpack.

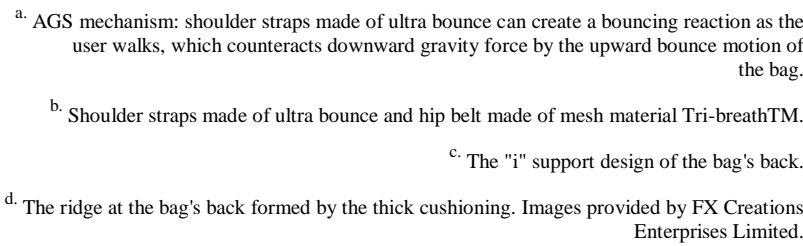

\section{B. Subjects}

Ten fit male freshman and sophomore students (age $19.9 \pm 0.7$ years; height $167.3 \pm 3.3 \mathrm{~cm}$; body mass $61.8 \pm 6.9$ $\mathrm{kg}$ ) from Guangzhou Sport University were recruited as subjects. They had no history of cardiovascular diseases, limb dysfunction or any bone, muscle and spine injuries. The participations were voluntary and all the subjects signed a written informed consent form. They were not allowed to undertake strenuous exercise within 24 hours before the experiments to keep them in good physical condition.

\section{Borg rating of perceived exertion}

Borg rating of perceived exertion (RPE) scale was adopted to measure the subjective physical fatigue of the test recipients during the 30-minute weight-bearing walking ("Table I"). Borg scale is a common way of measuring physical activity intensity level. It reflects the 
subject's total feeling of exertion that combines all sensations and feelings of physical stress, effort and fatigue [8]. It ranges from 6 to 20, where 6 means "no exertion at all" and 20 means "maximal exertion" [8] [9].

TABLE I. BORG RATING OF PERCEIVED EXERTION SCALE

\begin{tabular}{|c|c|c|c|c|c|c|c|c|c|c|c|c|c|c|c|}
\hline Scale & 6 & 7 & 8 & 9 & 10 & 11 & 12 & 13 & 14 & 15 & 16 & 17 & 18 & 19 & 20 \\
\hline $\begin{array}{c}\text { Level } \\
\text { of } \\
\text { exertion }\end{array}$ & $\begin{array}{c}\text { No } \\
\text { exertion } \\
\text { at all }\end{array}$ & $\begin{array}{c}\text { Extremely } \\
\text { light }\end{array}$ & $\begin{array}{c}\text { Very } \\
\text { light }\end{array}$ & Light & $\begin{array}{c}\text { Somewhat } \\
\text { light }\end{array}$ & & $\begin{array}{c}\text { Hard } \\
\text { (Heavy) }\end{array}$ & $\begin{array}{c}\text { Very } \\
\text { hard }\end{array}$ & $\begin{array}{c}\text { Extremely } \\
\text { hard }\end{array}$ & $\begin{array}{c}\text { Maximal } \\
\text { exertion }\end{array}$ \\
\hline
\end{tabular}

\section{Testing}

Two trials were conducted separately at an interval of 2 days. One was the test group in which the participants wore a backpack with AGS technology. The other was the control group in which the same participants wore a backpack with no AGS technology. The model number of the backpack with AGS technology in our study was SNA69723AGS. The backpack with no AGS technology used as the control group were also the products (model number YSD60605) provided by FX Creations Enterprises Limited. In each test, the subject was unaware of which type of backpack he was carrying on his back. In each trial, the subject wore minimal shorts and athletic shoes and had his upper bodies naked. He carried a loaded backpack (total mass: $5 \mathrm{~kg}$ ) on his back. The distance between the upper edge of backpack main body and the shoulder line of the subject was adjusted to 10 centimeters. At first, the subject stood still on the treadmill and was asked to give a score according to Borg RPE scale. It reflected his feeling of exertion caused by different types of backpacks in the initial static state and was recorded as the score at 0 minute. Then, the subject was required to perform a walking at a normal speed $(4.5 \mathrm{~km} / \mathrm{h})$ for 30 minutes on the treadmill ("Fig. 2"). During this process, the subject was asked about his fatigue feeling measured by Borg RPE scale every 5 minutes during the whole walking period.
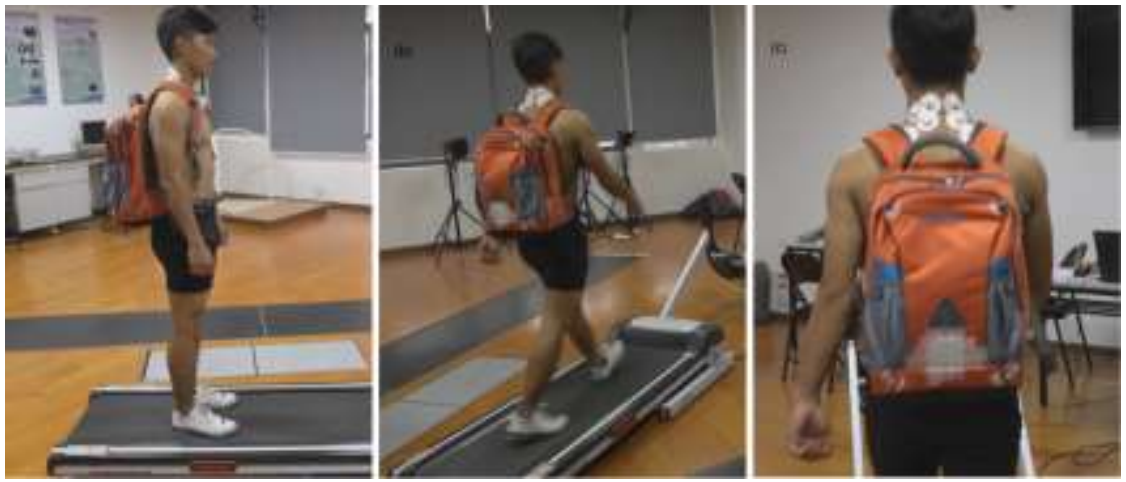

Fig. 2. Subject carrying a backpack in the process of experiment.

\section{RESULTS}

The result of subjective physical fatigue evaluation based on Borg RPE scale is shown in "Fig. 3". The scores at 0 minute reflected subjects' feeling of exertion caused by different types of backpacks in the initial static state. Six out of ten cases (subject 2, 3, 4, 6, 7 and 8 ) reported the same RPE scores between test group and control group, while three (subject 1, 5, 10) reported higher initial RPE scores in test group and one (subject 9) reported higher score in control group. As a. The static weight-bearing state of the subject at the time point of $0 \mathrm{~min}$ b. subject in walking stat the back view of the subject in walking state the walking began and continued, fatigue level in both test group and control group increased. Within the ten minutes after the walking began, three cases (subject 4, 8 and 10) reported the same fatigue levels between test group and control group. Five cases (subject 1, 2, 3, 5 and 6 ) reported higher fatigue levels in test group, while two cases (subject 7 and 9) reported higher scores in control group. In the final ten minutes of walking (20 min to $30 \mathrm{~min}$ ), the levels of fatigue in test group exceeded that in control group in eight cases (subject 1 , $2,3,4,7,8,9$ and 10). One subject (subject 5) reported 
higher fatigue level caused by AGS backpack at the 20th minute, and at the 25th and 30th minute, the levels of fatigue caused by AGS backpack and non-AGS backpack were the same. One subject (subject 6) reported the same levels of fatigue caused by AGS backpack and non-AGS backpack in the final ten minutes. The mean and standard deviation (std.) values of the RPE scores calculated respectively from AGS backpack group and non-AGS backpack group were

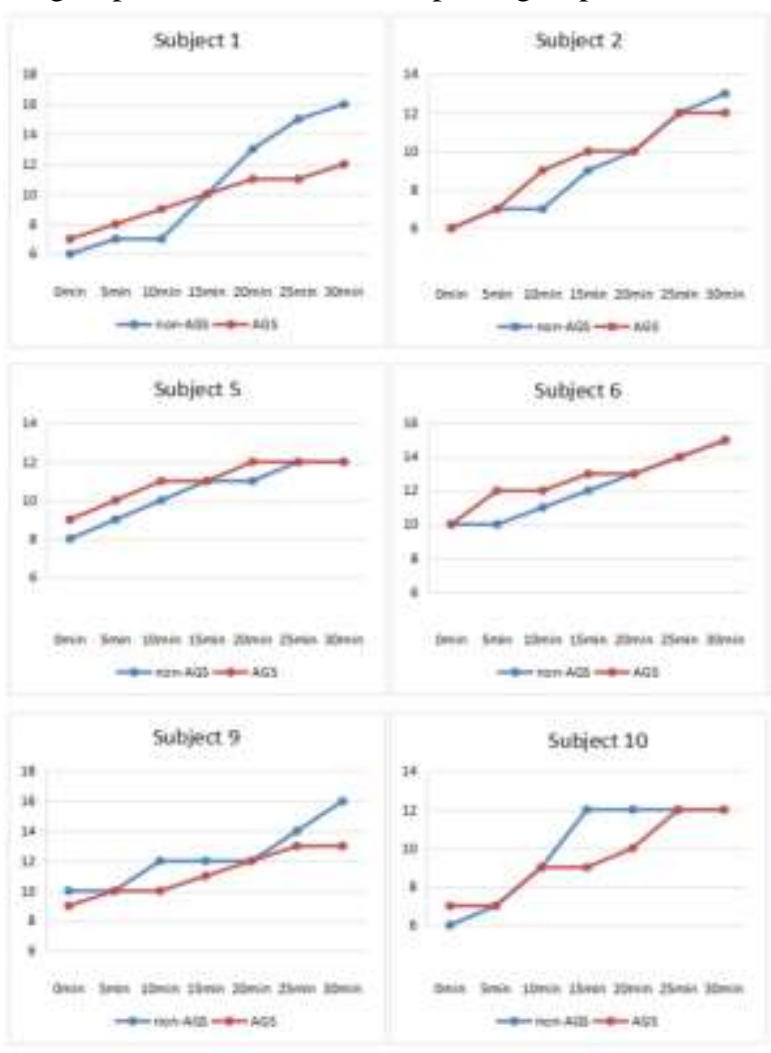

also compared. It can be seen that with the walking going on, the growth of mean RPE scores in AGS backpack group was slower than their counterparts in non-AGS backpack group. The std. values of RPE scores in test group were similar with those in control group initially, but had a decreasing tendency along with the experiment process. By contrast, the std. values of RPE scores in control group remained relatively high over time.

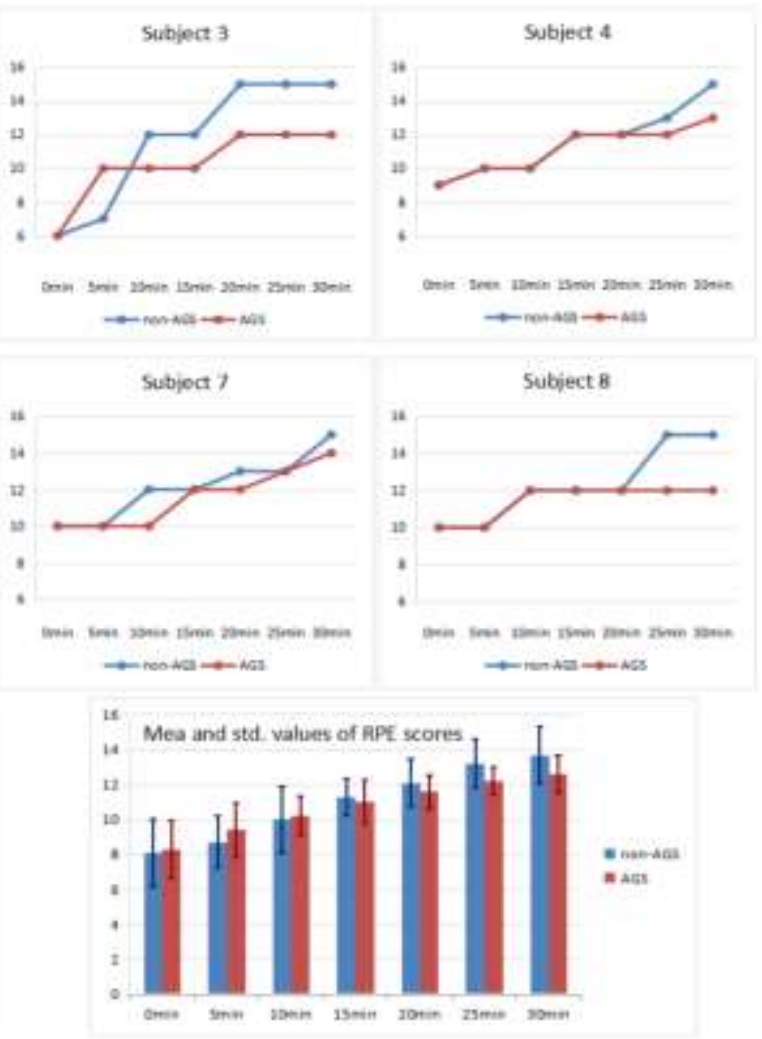

Fig. 3. Comparison between AGS backpack group and non-AGS backpack group on Borg RPE scores reported by the 10 subjects and on the mean and std. values of RPE scores calculated statistically from the 10 subjects at each time point.

\section{DISCUSSION}

The initial feeling of fatigue caused by AGS backpack and non-AGS backpack had no obvious difference. Along with the process of walking, in the case of subject 1, 3, 4, 8 and 9, the growth of RPE scores in non-AGS backpack group exceeded their counterparts in AGS backpack group. In the case of subject 2, 5, 6 and 7, the test group and the control group were almost at the same level during the entire process. In the case of subject 10, the RPE scores in test group and control group were very close to each other during the first ten minutes and the last five minutes, but existed a wide gap in the middle stage. It means that during the process of weight-bearing walking, the fatigue feelings exerted by different types of backpacks were different for more than half of the subjects.
AGS backpack group also displayed a slower growth of the mean RPE score and a decreasing tendency of RPE standard deviation during the weightbearing walking process. The larger std. values indicated that individual differences in the non-AGS backpack group on subjective fatigue feeling grew larger along with the progression of the weight-bearing walking. On the whole, AGS backpack can lead to a slower and more regular growth of subjective fatigue compared with non-AGS backpack.

\section{CONCLUSION}

For each subject, the test group experiment and the control group experiment were conducted respectively, and hence the results were closely related to the physical state of the subject at that time and also unavoidably influenced by the external environment. 
However, the variation trends of RPE scores can serve as reliable indicators of differences between AGS backpack group and non-AGS backpack group on subjective fatigue occurrence time and extent. It is concluded that the dynamic load triggered by anti gravity system is an effective measure to provide a burden reduction feeling to users and lead to a slower and more regular growth of subjective fatigue. In the future work, comparative experiments, in which more subjects of different gender and ages carrying backpacks loaded with different mass and walking for different lengths of time and at different speeds, will be conducted to further investigate the effects of AGS backpack on delaying or relieving subjective feeling of fatigue.

\section{Acknowledgment}

The study was funded by Guangdong Outstanding Young Teacher Training Program in Higher School (YQ201504).

\section{References}

[1] Gregory Denali Pro:additional photos of suspension [homepage on the Internet]. Moontrail, LLC; (C) 1997-2020 [cited 2020 Mar 8]. Available from: http://www.moontrail.com/gregory/denalipro/gregory-denali-pro-addsus.html

[2] 3D Tepex Frame [homepage on the Internet]. Moontrail, LLC; (C) 1998-2020 [cited 2020 Mar 8]. Available from: http://www.moontrail.com/granitegear-nimbusozone.php

[3] Y. Zhang, L. Zhang, "Mechanics on the shoulder pole," Mechnics in Engineering, vol. 24, pp. 76-78, May 2002.

[4] M. You, "A note to the mechanical principle in carrying with shoulder-pole," Mechnics in Engineering, vol. 33, pp. 87-89, August 2011.

[5] X. Qiu, "Does a shoulder pole save man's effort?" Mechnics in Engineering, vol. 40, pp. 108-111, February 2018.

[6] What is AGS? [homepage on the Internet] FX Creations International Ltd., Hong Kong, China; (C2020 [cited 2020 Mar 8]. Available from: http://fxcreations.com/What-is-AGS

[7] X. Yang, Y. Li, L. Guo, X. Sui, P. Ng, B. Kwan, "A comparative study of AGS and non-AGS backpacks on relieving fatigue of neck and back muscles," 2018 IEEE International Conference on Systems, Man, and Cybernetics (SMC), 2018

[8] G. Borg, Psychophysical scaling with applications in physical work and the perception of exertion, Scand. J. Work Environ. Health, vol.16, pp. 55-58, February 1990.

[9] N. Williams, The Borg Rating of Perceived Exertion (RPE) scale, Occup. Med., vol. 67, pp. 404-405, July 2017. 